The effects of viscoelastic fluid on kinesin transport

This article has been downloaded from IOPscience. Please scroll down to see the full text article.

2012 J. Phys.: Condens. Matter 24375103

(http://iopscience.iop.org/0953-8984/24/37/375103)

View the table of contents for this issue, or go to the journal homepage for more

Download details:

IP Address: 141.211.173.82

The article was downloaded on 26/06/2013 at 14:46

Please note that terms and conditions apply. 


\title{
The effects of viscoelastic fluid on kinesin transport
}

\author{
Woochul Nam and Bogdan I Epureanu \\ Department of Mechanical Engineering, University of Michigan, Ann Arbor, MI 48109-2125, USA \\ E-mail: epureanu@umich.edu
}

Received 10 April 2012, in final form 13 June 2012

Published 27 July 2012

Online at stacks.iop.org/JPhysCM/24/375103

\begin{abstract}
Kinesins are molecular motors which transport various cargoes in the cytoplasm of cells and are involved in cell division. Previous models for kinesins have only targeted their in vitro motion. Thus, their applicability is limited to kinesin moving in a fluid with low viscosity. However, highly viscoelastic fluids have considerable effects on the movement of kinesin. For example, the high viscosity modifies the relation between the load and the speed of kinesin. While the velocity of kinesin has a nonlinear dependence with respect to the load in environments with low viscosity, highly viscous forces change that behavior. Also, the elastic nature of the fluid changes the velocity of kinesin. The new mechanistic model described in this paper considers the viscoelasticity of the fluid using subdiffusion. The approach is based on a generalized Langevin equation and fractional Brownian motion. Results show that a single kinesin has a maximum velocity when the ratio between the viscosity and elasticity is about 0.5 . Additionally, the new model is able to capture the transient dynamics, which allows the prediction of the motion of kinesin under time varying loads.
\end{abstract}

(Some figures may appear in colour only in the online journal)

\section{Introduction}

Cells use various methods of mechanical transport. Among these, diffusion is a passive and effective means of transport in the presence of concentration gradients. However, if a cargo is too large or the required direction of a transport is against concentration gradients, then diffusion is not enough. For this situation, molecular motors are necessary to move cargoes in the cell. Molecular motors have important functions in various processes such as muscle contraction, cell division, vesicle transport, operation of flagella and cilia, and DNA metabolism [1-4]. The cytoskeletal motors (e.g., kinesin and dynein) walk along microtubules by converting chemical energy into mechanical movement. This paper focuses on kinesin-1 which moves toward the plus end of microtubules. This motor protein (referred to simply as kinesin) makes use of energy from the hydrolysis of adenosine triphosphate (ATP) to carry various vesicles and organelles to their destinations. Kinesin is also involved in assembling spindles and segregating chromosome in mitosis [5-7]. Due to advances in motility assays (e.g., bead assays), laser trapping systems, and optical technology, the motion of a single molecule can be observed experimentally in vitro.

Kinesin has two head domains and walks in a hand-overhand fashion by generating forces of up to $6 \mathrm{pN}[8,9]$. A step of $16 \mathrm{~nm}$ length of each head domain results in a discrete $8 \mathrm{~nm}$ advance of the cargo pulled by kinesin. The maximum velocity of kinesin is about $800 \mathrm{~nm} \mathrm{~s}^{-1}$. This velocity varies with the ATP concentration and the load [10-14]. Several models for kinesin have been created based on experimental data [15-24]. They can successfully describe the motion of kinesin in vitro. However, most models have difficulties predicting the dynamics of kinesin in other conditions. First, several molecular motors cooperate to pull a common cargo, and the number of kinesin for one cargo rapidly changes over time [25]. In addition, molecular motors switch their tracks [26]. These changes cause continuous transient motion. Second, the intracellular fluid is different from water (used for in vitro experiments). It is much more viscous than water and also has elasticity. The large complex modulus of fluid is expected to be a significant factor affecting the motion of kinesin. Holzwarth et al [27] calculated the required force for 
kinesin to pull a cargo in the cytoplasm. With the complex modulus data of a COS7 cell, they concluded that a very strong force $(\sim 75 \mathrm{pN})$ is needed to transport a bead of radius $0.1 \mu \mathrm{m}$. However, their results are based on the assumption that the motion of kinesin in vivo is the same as the motion in vitro. In addition, their method applies only to steady state motion. The velocity of kinesin in cellular fluids and in artificial viscoelastic fluids is observed by [28-30]. They also estimated the velocity in cellular fluid by using the force-velocity curve of kinesin in vitro and the viscoelastic properties that correspond to a very low frequency motion. Their calculation showed a good match between numerical and experimental results. However, their method is sensitive to the selection of the value chosen for the low frequency, and the effects of elasticity remain unclear. Therefore, the goal of this paper is to develop a model which is capable of describing both transient and steady state motions. The model also considers the viscoelasticity of fluid so that the effects of viscosity and elasticity on kinesin can be revealed. The transient dynamics is particularly important in a highly viscoelastic fluid because kinesin requires a relatively long time before reaching its equilibrium configuration (i.e. $8 \mathrm{~nm}$ advance of a cargo takes much longer than the time in low viscous fluid). Thus, previous models which do not consider the transient motion are insufficient for modeling the motion of kinesin in a viscoelastic fluid.

To capture both transient and steady state dynamics, components of kinesin are regarded as linear elastic elements in our model. We also assume that the diffusion of a free head to the next binding site is much faster than the time needed for one cycle of the chemical process to complete (i.e., the dwell time). Thus, the diffusion time is negligible. The interactions between motor heads and ATP/ADP are modeled as Michaelis-Menten kinetics, and the effects of forces on the rates of chemical kinetics are accounted for by using the Arrhenius equation [31]. Theoretical studies provide models for the dynamics of a sphere moving in a viscoelastic fluid [32-36]. One of the fundamental assumptions of these studies is that the fluid behaves as if it contains small, linearly elastic spheres which account for the elasticity of the fluid. This assumption is feasible if the sphere of interest is very large compared to other particles in the fluid or the particles have the shape of sphere. However, the size of a motor protein and its cargo is comparable to other particles in the cytoplasm and cells have particles whose shapes are not sphere. Furthermore, particles present in vivo are not purely elastic. They have viscosity as well as elasticity. Thus, their models cannot be simply extended to the motion of kinesin in a viscoelastic fluid. Hence, newly developed analysis tools from microrheology have to be used for this study. Subdiffusion, generalized Langevin equation (GLE), generalized Stokes Einstein relation (GSE), and fractional Brownian motion (FBM) are some of the main concepts which can be used to better understand the effect of viscoelastic fluid on the motion of kinesin [37-41]

The GLE is used with some modifications in this work to predict the motion of a spherical cargo in the cytoplasm. This equation is an improved version of the Langevin equation which governs the motion of a particle in a purely viscous fluid (used for in vitro models). Thermal fluctuations of a cargo in a purely viscous fluid have a white noise distribution (normal diffusion) because there is no temporal correlation in the fluctuations. Thus, the resulting mean square displacement (MSD) of the cargo is proportional to $t$ (time). However, the cytoplasm exhibits subdiffusion where the MSD due to thermal fluctuations is proportional to $t^{\alpha}$, where $\alpha$ is a constant $(0<\alpha<1)$. This MSD results in a complex modulus which has fractional slopes over frequency on a log scale [42]. In contrast, a system composed of linear elastic and viscous elements has integer slopes. This indicates that subdiffusion cannot be accurately modeled as a system composed of linear springs and dashpots. Therefore, the motion of a particle in cellular fluid has to be described using the GLE. Note that subdiffusion results from temporal correlations in thermal fluctuations. This means that the system dynamics depends not only on current states but also on past states. The GLE accounts for this behavior by using a convolution term of velocities and a memory function.

To study kinesin in a viscoelastic fluid, it is necessary to start by first measuring the complex modulus of the fluid. Since the properties of a fluid are measured by interactions between the particles in the fluid and a measurement probe, the size of the probe is an important parameter. The size of kinesin cargoes is of the order of $1 \mu \mathrm{m}$, so microrheology has to be employed. In microrheology, there are two ways of measuring the complex modulus of a fluid [43]. The active method is to apply forces to a probe particle from the outside of the system by using an optical trap or a magnetic tweezer. The passive method uses thermal fluctuations as excitation mechanism. In this method, a particle that already exists in the fluid is used as a probe to minimize the intervention into the original system. The MSD of the probe particle is observed over time using video tracking or laser tracking systems. The GSE provides a method to calculate the complex modulus from the measured MSD by using the GLE. The Fourier transform of the GLE establishes the relation between the complex modulus and the MSD in the frequency domain Finally, the experimental MSD data is transformed to the frequency domain and substituted into the GLE [44]. This method is limited by the distance the particle is allowed to travel. In a highly dense fluid, the area in which a particle can travel by thermal fluctuations is very small compared to its size. It is also possible that the particle becomes trapped in networks of long polymers and cannot escape without external forces. It is reported that the MSD of a particle in a viscoelastic fluid flattens after a long time [39, 44-46]. This observation supports the idea that the particle becomes trapped by polymers. Thus, the features of a motion of a particle that travels over a long distance in a fluid cannot be found passively. Nevertheless, this passive method is used frequently because it is less invasive compared to the active method where the external force can have effects not only on the probe particle but also on other particles in the fluid.

Generally, the complex modulus of a viscoelastic fluid over frequency can correspond to a power law behavior, and the power can vary over the frequency of excitation. In this 
(a)

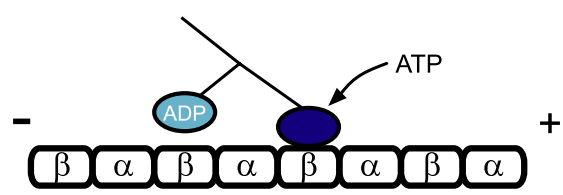

(b)

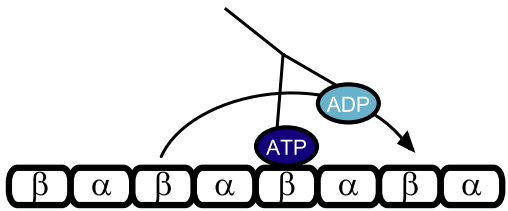

(c)

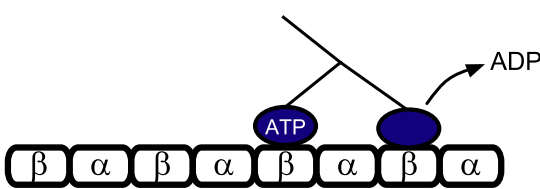

Strongly bound

Weakly bound or free

(d)

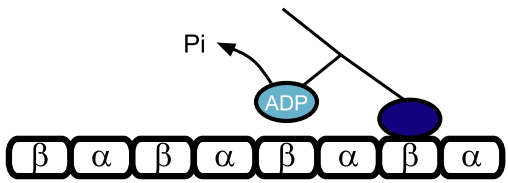

Figure 1. The mechanochemical cycle of kinesin. The plus and minus signs denote the polarity of microtubule. (a), (b) ATP binding to the leading head results in a conformational change in the molecule. The trailing head moves to the next binding site by relaxing the stresses caused by the conformational change and by Brownian motion. (c) The free head strongly binds to the new binding site by dissociating ADP. (d) ATP is hydrolyzed into ADP and phosphate. Then, the trailing head become weakly bound after releasing phosphate. The cycle repeats starting with (a).

paper, however, the complex modulus corresponding to a power law with a constant power is considered. For example, water mixed with $2 \mathrm{mg} \mathrm{ml}^{-1}$ of xanthan follows such a power law [28]. This assumption is reasonable for some cellular fluids also. For example, the cytoplasms of PC12 and NT2 cells have complex modulus whose powers are approximately constant over a wide range of frequencies $[29,47]$. To simplify the memory function in the GLE, the complex modulus is assumed to have a single constant fraction number in this paper. This assumption leads to a FBM which comes from a single fractional Gaussian noise [37]. The FBM provides a simple memory function which is proportional to $t^{-\alpha}$ (where $\alpha$ characterizes the ratio between viscosity and elasticity). A fluid characterized by a small $\alpha$ has higher elasticity and lower viscosity than a fluid characterized by a large $\alpha$.

Two stochastic factors are inherent in the motion of kinesin. One comes from the thermal fluctuations, and the other is related to the chemical reaction between ATP and the motor heads. The model used here is deterministic and provides predictions for the mean behavior of kinesin for both transient and steady state motions. A deterministic model is used because it has significantly higher computational efficiency than any other stochastic computational model.

\section{Description of the model}

The model of kinesin moving in a viscoelastic fluid takes into account two main processes: the walking motion of kinesin, and the forces created by the viscoelastic fluid. The first process is modeled based on the mechanistic mathematical formulation of [48-50]. The second process, viscoelastic effects in a subdiffusional environment, is implemented using the FBM.

\subsection{Walking of kinesin}

A kinesin molecule is assumed to be composed of two heads, two neck linkers, and one neck. Each head is connected to the neck by a neck linker, and the cargo is linked to the neck via a cargo linker. Kinesin walks toward the plus end of a microtubule by a repeated mechanochemical cycle, as depicted in figure 1. Each linker is assumed to be a linearly elastic element. The linkers are also assumed to have tether behavior. They resist tensile forces, but do not carry compressive loads. Thus, the dynamics is not linear.

The forces between the neck and the cargo are determined by three cases of interaction between the cargo and the kinesin molecule. Figure 2 shows those cases.

The force exerted by the neck linker is depicted in figure 3. The backward head is free or weakly bound in most states of its mechanochemical cycle. Also, there is no evidence that a cargo moves significantly during the time when the kinesin changes from the state where both heads strongly bound to the state where only the forward head is strongly bound. Thus, the model assumes that a load on the cargo linker is transferred to the neck linker of the forward head only, and the load does not act on the other neck linker.

Based on the interaction and forces depicted in figures 2 and 3 , the relation between the position of the neck and cargo and the resulting forces can be expressed as follows. 


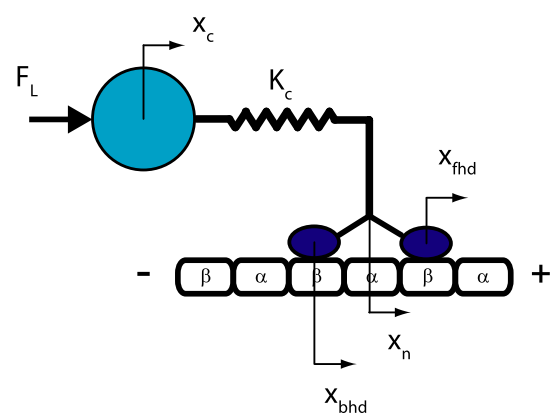

(a)

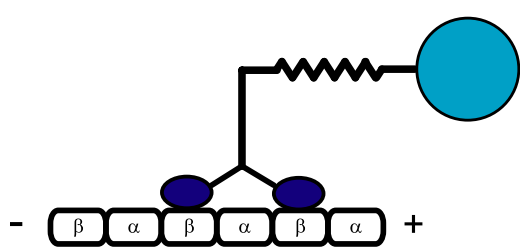

(b)

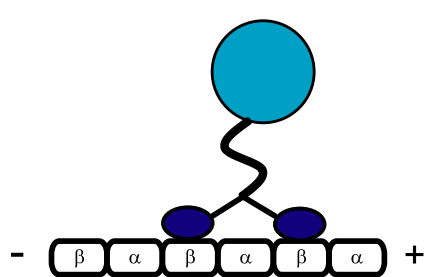

(c)

Figure 2. Interactions between the cargo and a kinesin. (a) The kinesin pulls the cargo. (b) The cargo pulls the kinesin. (c) The cargo linker is slack.

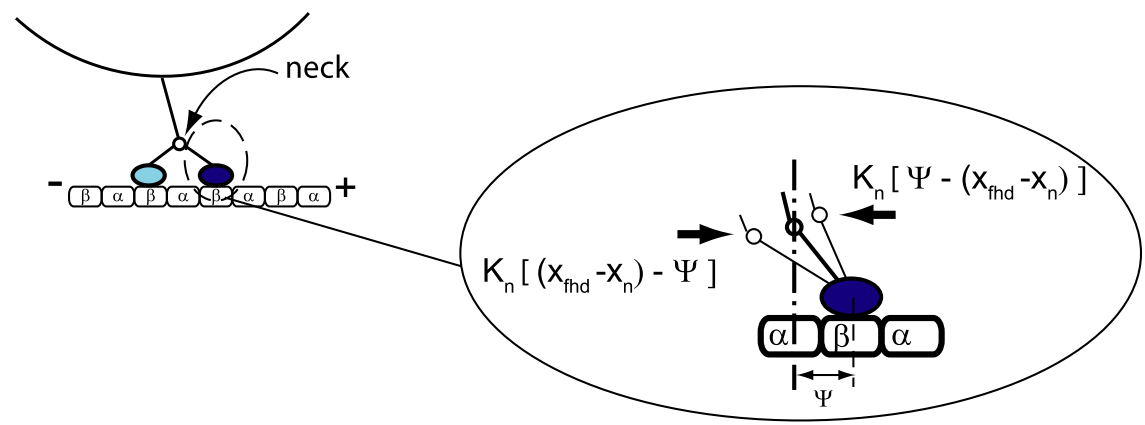

Figure 3. Forces acting on the neck by the neck linker of the forward head. The equilibrium position of the neck is shown by the bold dot line. The arrows represent the forces exerted on neck by the neck linkers when the neck is not at its the equilibrium position. The directions of the arrows/forces are toward the equilibrium position.

If a kinesin pulls the cargo (i.e., $\left.x_{\mathrm{n}}(t) \geq x_{\mathrm{c}}(t)+L_{\mathrm{c}}\right)$, then

$$
\begin{aligned}
K_{\mathrm{c}}\left[x_{\mathrm{n}}(t)-x_{\mathrm{c}}(t)-L_{\mathrm{c}}\right] & =K_{\mathrm{n}}\left[\left(x_{\mathrm{fhd}}-x_{\mathrm{n}}(t)\right)-\Psi\right] \\
\text { and } \quad F_{\mathrm{c}}(t) & =F_{\mathrm{L}}(t)+K_{\mathrm{c}}\left[x_{\mathrm{n}}(t)-x_{\mathrm{c}}(t)-L_{\mathrm{c}}\right] .
\end{aligned}
$$

If the cargo pulls a kinesin (i.e., $x_{\mathrm{n}}(t) \leq x_{\mathrm{c}}(t)-L_{\mathrm{c}}$ ), then

$$
\begin{gathered}
K_{\mathrm{c}}\left[x_{\mathrm{c}}(t)-x_{\mathrm{n}}(t)-L_{\mathrm{c}}\right]=\left[\Psi-\left(x_{\mathrm{fhd}}-x_{\mathrm{n}}(t)\right)\right] \\
\text { and } \quad F_{\mathrm{c}}(t)=F_{\mathrm{L}}(t)+K_{\mathrm{c}}\left[x_{\mathrm{n}}(t)-x_{\mathrm{c}}(t)+L_{\mathrm{c}}\right] .
\end{gathered}
$$

Finally, if the cargo linker is slack (i.e., $x_{\mathrm{c}}(t)-L_{\mathrm{c}}<x_{\mathrm{n}}(t)<$ $\left.x_{\mathrm{c}}(t)+L_{\mathrm{c}}\right)$, then

$$
\begin{aligned}
0 & =K_{\mathrm{n}}\left[\left(x_{\mathrm{fhd}}-x_{\mathrm{n}}(t)\right)-\Psi\right] \quad \text { and } \\
F_{\mathrm{c}}(t) & =F_{\mathrm{L}}(t) .
\end{aligned}
$$

Variables $x_{\mathrm{fhd}}, x_{\mathrm{bhd}}, x_{\mathrm{n}}$ and $x_{\mathrm{c}}$ in equation (1) denote the positions of the forward head, the backward head, the neck and the cargo. $\Psi$ is the distance between the forward head and neck when the internal strain in the neck linker is zero. The value of $\Psi$ is chosen as $4 \mathrm{~nm}$. $K_{\mathrm{c}}$ and $K_{\mathrm{n}}$ are the stiffness of the cargo linker and neck linker, and $F_{\mathrm{L}}$ is the external load. $L_{\mathrm{c}}$ is the unstretched length of the cargo linker, and $F_{\mathrm{c}}$ is the resultant force on the cargo.

Each head experiences the attachment of ATP and its hydrolysis at every step. This chemical reaction is described by a Michaelis-Menten kinetics as

$$
\begin{aligned}
E+ & S(K+A T P) \rightleftharpoons{ }_{k_{1 b}}^{k_{1 f}} E S(K \mathrm{ATP}) \rightarrow^{k_{2 f}} E \\
& +P(K+\mathrm{ADP}+P i),
\end{aligned}
$$

where $E, S, P$ and $K$ denote enzyme, substrate, product, and kinesin, respectively.

By using single molecule Michaelis-Menten equations [51], the rate of these two reactions can be calculated as

$$
\frac{\mathrm{d} \rho}{\mathrm{d} t}= \pm \frac{2 k_{2 f}[\mathrm{ATP}]}{K_{\mathrm{M}}+[\mathrm{ATP}]}
$$

where $K_{\mathrm{M}}=\frac{k_{1 b}+k_{2 f}}{k_{1 f}}$. The variable $\rho$ is a state variable whose time rate of change captures the average velocity of the chemical process. The value of $\rho$ becomes 1 (or -1 ) when the chemical reaction is complete. Then, $\rho$ starts to decrease (or increase) to reach -1 (or 1 ). The values of 1 and -1 are not physical but just reference values to capture the instants when a cycle of the chemical reaction completes. A more detailed explanation is given in [48].

This chemical reaction depends on the force that acts on the motor heads because the force affects the geometry of the heads. A reaction rate constant depends exponentially on the energy difference between states [31]. Thus, the effects of the force on the chemical kinetics can be included in the dissociation rate constant of ATP by accounting for the strains in the protein (i.e., changes in the geometry of the protein) due to forces. That can be expressed as

$$
k_{1 b}=k_{1 b, 0} \exp \left[\frac{\frac{1}{2} \kappa\left|x_{\mathrm{fhd}}-x_{\mathrm{n}}-\Phi_{\mathrm{c}}\right|^{2}}{k_{\mathrm{B}} T}\right],
$$


where $x_{\text {fhd }}$ and $x_{\mathrm{n}}$ denote the positions of the forward head and the neck, and $\Phi_{\mathrm{c}}$ and $\kappa$ are constants of the model. This equation captures the fact that the rate constant $k_{1 b}$ is minimum $\left(k_{1 b}=k_{1 b, 0}\right)$ when the protein is in equilibrium, i.e. when $x_{\text {fhd }}-x_{\mathrm{n}}=\Phi_{\mathrm{c}}$.

The motion of kinesin involves two time scales. The time needed for one cycle of the chemical process to complete is referred to as the dwell time. The diffusion time is the required time for a head which just become free to step to the next binding site by Brownian motion and by relaxing the stresses due to the conformational change. This diffusion time is much shorter than the dwell time in vitro [52]. For a higher viscous fluid, the diffusion time increases, but the increase is not large enough to make the diffusion time comparable to the dwell time. Since the size of a kinesin neck and that of a head are very small compared to polymers and other particles in a cell, it is plausible to assume that the diffusion time is very short even in highly viscous environments (e.g., in vivo). Furthermore, the cargo also experiences increased drag forces in fluids with increased viscosity. That causes the chemical reaction to slow down. For nano-size particles, the viscosity that the particles experience increases exponentially over their sizes [53, 54]. As the cargo is larger than the kinesin heads and neck, the effect of the high viscosity on the chemical reaction is stronger than its effect on the diffusion time. The supplementary material of Gennerich and Schild [55] provides further evidence of this assumption using the experimental data of [25]. Thus, we can assume that diffusion occurs approximately instantaneously.

\subsection{Effects of viscoelasticity}

2.2.1. Approximate FBM. Due to the small size of the kinesin molecule, direct effects of the fluid on it can be neglected. However, the viscoelastic effects on the cargo are important. They are described with the GLE. One obtains

$$
m_{\mathrm{c}} \frac{\mathrm{d} v_{\mathrm{c}}(t)}{\mathrm{d} t}=-\zeta \int_{0}^{t} v_{\mathrm{c}}(\tau) H(t-\tau) \mathrm{d} \tau+F_{\mathrm{th}}(t)+F(t),
$$

where $H(t)$ is the memory function, $F_{\mathrm{th}}(t)$ is the force caused by thermal fluctuations, $\zeta$ is a constant which represents the intensity of the viscoelastic forces, and $m_{\mathrm{c}}$ and $v_{\mathrm{c}}$ are the mass and velocity of the cargo. For a purely viscous fluid, $\zeta$ is the usual damping coefficient (and has a value of $6 \pi r \eta$ for a sphere of radius $r$ moving in the fluid).

The force $F_{\mathrm{th}}(t)$ is related to the memory function by $\left\langle F_{\mathrm{th}}(t) F_{\mathrm{th}}\left(t^{\prime}\right)\right\rangle=k_{\mathrm{B}} T \zeta H\left(t-t^{\prime}\right)$, where $\langle\cdot\rangle$ represents the mean over time. This force is not included in our deterministic model because its mean value is zero.

The objective of the model is to predict the motion for a given force. However, the original GLE in equation (5) has an implicit form for the velocity. To solve this problem, the original GLE is converted into an explicit form for the displacement. Also, the inertial term is neglected because the size of the cargo is of the order of $1 \mu \mathrm{m}$ and thus, its inertia is very small compared to other forces. The thermal fluctuations of the cargo are very fast compared to the time scale of the walking of kinesin. Thus, most of the thermal forces are assumed to cancel out rapidly, and their effects on the kinesin molecule is considered negligible. With these assumptions, one obtains

$$
x_{\mathrm{c}}(t)=x_{\mathrm{c}}(0)+\frac{1}{\zeta} \int_{0}^{t} D(t-\tau) F_{\mathrm{c}}(\tau) \mathrm{d} \tau,
$$

where $D(t)=\mathcal{L}^{-1}\left[\frac{1}{s \mathcal{L}\{H(t)\}}\right]$, with $\mathcal{L}$ and $\mathcal{L}^{-1}$ representing the Laplace transform and inverse Laplace transform.

Experiments on certain neuron cells have revealed that the complex modulus of the intracellular fluid approximately follows a FBM with a single fractional number $\alpha \approx 0.8$ (where $\alpha$ characterizes the ratio between viscosity and elasticity) [29]. Substituting in equation (6) the expression for the memory function of FBM with a single $\alpha$, one obtains

$$
x_{\mathrm{c}}(t)=x_{\mathrm{c}}(0)+\frac{1}{\zeta} \frac{\Gamma(\alpha)}{\Gamma(3-\alpha)} \int_{0}^{t} M(t-\tau) F_{\mathrm{c}}(\tau) \mathrm{d} \tau,
$$

where $M(\tau)=|\tau|^{\alpha-1}$ is the memory function of this modified GLE, and $\Gamma$ is the gamma function [37]. Note that $\zeta$ is related to the magnitude of complex modulus of a viscoelastic fluid (see equations (11) and (12)).

The current position and force on the cargo can be calculated using equations (1) and (7). However, equation (7) involves high computational costs due to two issues related to the convolution term.

First, the numerical calculation of the convolution term is difficult because the function, $M(t-\tau)=|t-\tau|^{\alpha-1}$, is infinite at $\tau=t$. To address this issue, we approximate the force $F_{\mathrm{c}}$ on the cargo as linearly varying in time between two time instances which are very close, i.e. between $\tau=t-\varepsilon$ and $t$. This approximation enables the integration between $t-\varepsilon$ and $t$ as follows

$$
\begin{aligned}
& \int_{t-\varepsilon}^{t} M(t-\tau) F_{\mathrm{c}}(\tau) \mathrm{d} \tau=\int_{t-\varepsilon}^{t}(t-\tau)^{\alpha-1} F_{\mathrm{c}}(\tau) \mathrm{d} \tau \\
& \simeq \int_{t-\varepsilon}^{t}(t-\tau)^{\alpha-1}\left[F_{\mathrm{c}}(t)+\frac{F_{\mathrm{c}}(t)-F_{\mathrm{c}}(t-\varepsilon)}{\varepsilon}(\tau-t)\right] \mathrm{d} \tau \\
& =F_{\mathrm{c}}(t) \frac{1}{\alpha} \varepsilon^{\alpha}+\frac{F_{\mathrm{c}}(t)-F_{\mathrm{c}}(t-\varepsilon)}{\varepsilon} \frac{1}{\alpha+1}\left(-\varepsilon^{\alpha+1}\right)
\end{aligned}
$$

Second, the convolution term in equation (7) has to be computed at every time step, and that increases the calculation time significantly. To address this issue, we developed a new method which approximates the memory function. Specifically, the rate of change of the memory function is proportional to $(t-\tau)^{\alpha-2}$, and this value is small in the region where $\tau$ is not close to $t$. So, the memory function can be assumed to be piece-wise linear in that region (figure 4). Then, the calculation of the convolution integral can be simplified as follows

$$
\begin{aligned}
\int_{0}^{t} M(t-\tau) F_{\mathrm{c}}(\tau) \mathrm{d} \tau= & \int_{0}^{t}(t-\tau)^{\alpha-1} F_{\mathrm{c}}(\tau) \mathrm{d} \tau \\
\approx & {\left[\int_{0}^{u_{1}}\left(a_{1} \tau+b_{1}\right) F_{\mathrm{c}}(\tau) \mathrm{d} \tau\right.} \\
& +\int_{u_{1}}^{u_{2}}\left(a_{2} \tau+b_{2}\right) F_{\mathrm{c}}(\tau) \mathrm{d} \tau+\cdots \\
& \left.+\int_{u_{N-1}}^{u_{\mathrm{N}}}\left(a_{\mathrm{N}} \tau+b_{\mathrm{N}}\right) F_{\mathrm{c}}(\tau) \mathrm{d} \tau\right]
\end{aligned}
$$




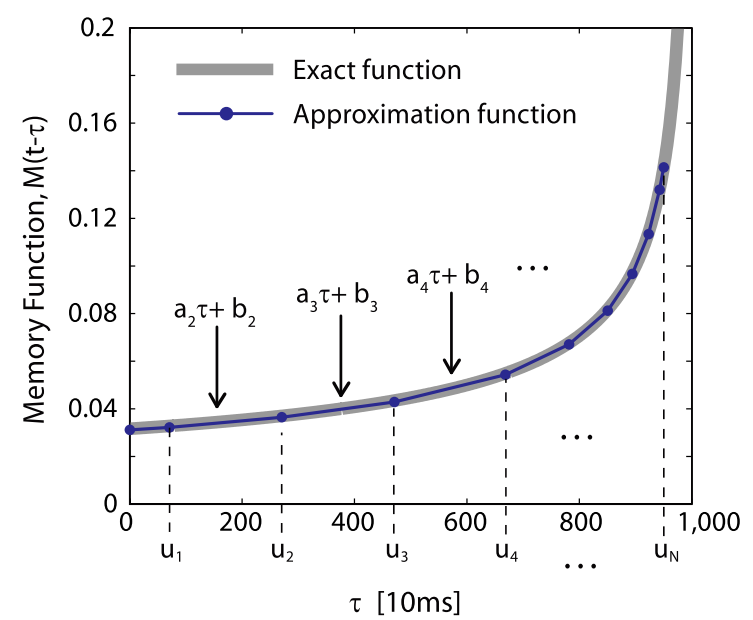

Figure 4. Approximation of the memory function, $M(t-\tau)$. The memory function for $\alpha=0.5$ and $t=1000(\times 10 \mathrm{~ms})$ is shown. $a_{i} \tau+b_{i}$ indicates the equation for the $i$ th linear approximation.

$$
\begin{aligned}
& +\int_{u_{\mathrm{N}}}^{t}(t-\tau)^{\alpha-1} F_{\mathrm{c}}(\tau) \mathrm{d} \tau \\
= & \sum_{i=0}^{i=N}\left[a_{i}\left(U\left(u_{i+1}\right)-U\left(u_{i}\right)\right)+b_{i}\left(V\left(u_{i+1}\right)-V\left(u_{i}\right)\right)\right] \\
& +\int_{u_{\mathrm{N}}}^{t}(t-\tau)^{\alpha-1} F_{\mathrm{c}}(\tau) \mathrm{d} \tau,
\end{aligned}
$$

where $u_{i}$ are time instances between 0 and $t$, while $a_{i}$ and $b_{i}$ are slopes and $y$-intercepts of each line, as shown in figure 4. The functions $U\left(u_{i}\right)$ and $V\left(u_{i}\right)$ are defined as $\int_{0}^{u_{i}} \tau F_{\mathrm{c}}(\tau) \mathrm{d} \tau$ and $\int_{0}^{u_{i}} F_{\mathrm{c}}(\tau) \mathrm{d} \tau$.

While all past data is needed to compute the convolution term at the current instant, the simplified computation of equation (9) requires discrete data only at few time instances $u_{i}$ and the calculation of a single integral (only from $u_{\mathrm{N}}$ to $t$ instead of one from 0 to $t$ ). Hence, this approach reduces the calculation time significantly.

Finally, the position of cargo is calculated by substituting equations (8) and (9) into (7). One obtains

$$
\begin{aligned}
x_{\mathrm{c}}(t)= & x_{\mathrm{c}}(0)+\frac{1}{\zeta} \frac{\Gamma(\alpha)}{\Gamma(3-\alpha)}\left[\sum _ { i = 0 } ^ { i = N } \left\{a _ { i } \left(U\left(u_{i+1}\right)\right.\right.\right. \\
& \left.\left.-U\left(u_{i}\right)\right)+b_{i}\left(V\left(u_{i+1}\right)-V\left(u_{i}\right)\right)\right\} \\
& +\int_{u_{\mathrm{N}}}^{t-\varepsilon}(t-\tau)^{\alpha-1} F_{\mathrm{c}}(\tau) \mathrm{d} \tau+F_{\mathrm{c}}(t) \frac{1}{\alpha} \varepsilon^{\alpha} \\
& \left.+\frac{F_{\mathrm{c}}(t)-F_{\mathrm{c}}(t-\varepsilon)}{\varepsilon} \frac{1}{\alpha+1}\left(-\varepsilon^{\alpha+1}\right)\right]
\end{aligned}
$$

\subsubsection{Relation between the FBM and the complex modulus.}

Because a viscoelastic fluid allows energy storage and loss, its modulus contains both real and imaginary part. In the frequency domain, the complex modulus $G$ is expressed as $G(f)=G^{\prime}(f)+\mathrm{j} G^{\prime \prime}(f)$, where $f$ represents frequency. The value of the real and imaginary part are determined by MSD and $\alpha$ as follows [44]

$$
\begin{aligned}
|G(f)| & =\frac{k_{\mathrm{B}} T}{\pi r \mathrm{MSD}(t=1 / f) \Gamma[1+\alpha(f)]} \\
G^{\prime}(f) & =|G(f)| \cos (\pi \alpha(f) / 2) \\
G^{\prime \prime}(f) & =|G(f)| \sin (\pi \alpha(f) / 2) .
\end{aligned}
$$

where $r$ is radius of the interesting sphere in the fluid. The phase of the complex modulus can be expressed with $\alpha$. An $\alpha$ value of 0 (or 1) corresponds to a fluid which is purely elastic (or purely viscous). For the fluid characterized by a single fractional number, a small $\alpha$ indicates that the fluid has higher elasticity and lower viscosity and vice versa. $G_{\mathrm{r}}$ and $f_{\mathrm{r}}$ are used to determine the magnitude of the complex modulus. The magnitude at a certain frequency depends on the values of MSD and $\alpha$ at that frequency. Also, MSD depends on $\alpha$ and $\zeta$ [37] as follows

$$
\operatorname{MSD}(t)=\frac{k_{\mathrm{B}} T}{\zeta} \frac{\sin (\alpha \pi)}{\pi(1-\alpha / 2)(1-\alpha) \alpha} t^{\alpha} .
$$

Thus, $\zeta$ in equation (7) is determined using equations (11) and (12).

The parameter $f_{\mathrm{r}}$ is a reference frequency (a value around $\left.0.01 \mathrm{~s}^{-1}\right)$. The parameter $G_{\mathrm{r}}$ is the ratio of $G^{\prime \prime}$ for water and $G^{\prime \prime}$ for the fluid of interest at the reference frequency $f=f_{\mathrm{r}}$.

If viscosity and elasticity are caused by independent elements like a spring and a dashpot, then one can define viscosity and elasticity separately. Then, it is easy to observe their effects separately. For a fluid of a single fractional number, however, viscosity and elasticity are coupled by the fractional number. Therefore, it is impossible to change one property while the other is fixed. Either viscosity or elasticity can be the reference for the magnitude of viscoelasticity. If the elasticity is used as the reference value, then a fluid characterized by a large $\alpha$ has higher elasticity and higher viscosity than a fluid characterized by a small $\alpha$. Then, the comparison of the motion of kinesin over $\alpha$ can become unclear because both properties increase with $\alpha$. Thus, using $G^{\prime \prime}$ as a reference value of viscosity can lead to more clear comparisons for various values of $\alpha$.

$G^{\prime \prime}$ has a slope of $\alpha$ over frequency in a log scale. Thus, the ratio between $G^{\prime \prime}$ and $G_{\text {water }}^{\prime \prime}$ changes over frequency. The reference frequency $f_{\mathrm{r}}$ deals with this issue. When $f>f_{\mathrm{r}}$, the smaller $\alpha$ is, the lower the viscosity of the fluid is. When $f<f_{\mathrm{r}}$, the relation between $\alpha$ and viscosity is reversed. As we want a fluid of small $\alpha$ to have low viscosity over a wide range of frequencies, $f_{\mathrm{r}}$ is chosen to have a low value $\left(f_{\mathrm{r}}=0.01 \mathrm{~s}^{-1}\right)$, as shown in figure 5 .

\subsection{Calculation procedure}

To compute the motion using our model, the variables which determine the mechanochemical motion of kinesin are updated in time. During each time step $\Delta t$ (which typically is a small fraction of the dwell time) the cargo and neck interact as given by the mechanical governing equations (equations (1) and (10)). The (past) data is stored to evaluate the memory effects due to the viscoelastic fluid. At the current time 


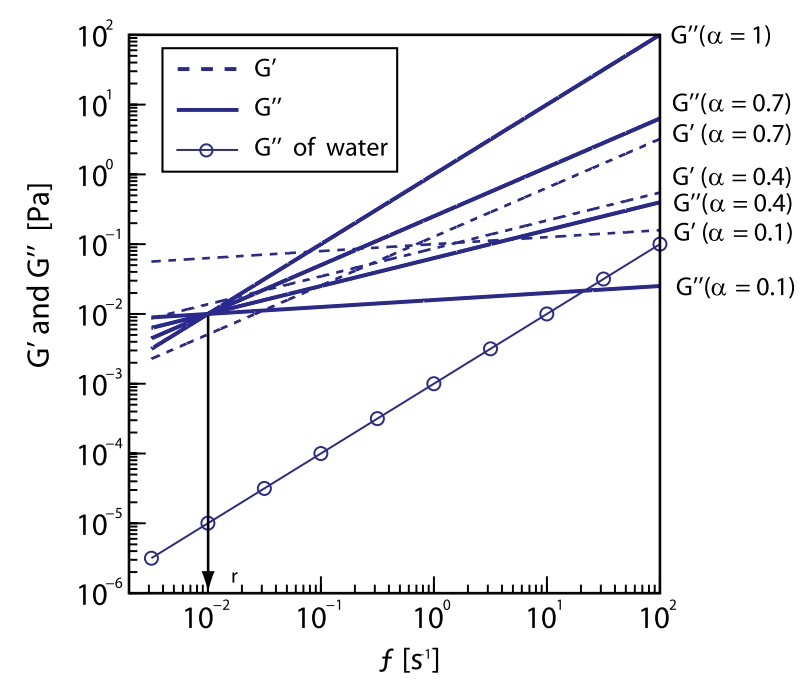

Figure 5. Complex modulus for $G_{\mathrm{r}}=1000$, and $\alpha=0.1,0.4,0.7$ and 1.

instant $t$, the required (past) data are the value of $F_{\mathrm{c}}(\tau)$ for all $\tau$ from $u_{\mathrm{N}}$ to $t-\Delta t, x_{\mathrm{fhd}}(t-\Delta t), x_{\mathrm{bhd}}(t-\Delta t), x_{\mathrm{n}}(t-\Delta t)$, $x_{\mathrm{c}}(t-\Delta t), \rho, \int_{0}^{t-\Delta t} F_{\mathrm{c}} \mathrm{d} \tau$, and $\int_{0}^{t-\Delta t} \tau F_{\mathrm{c}} \mathrm{d} \tau$. Here, $\Delta t$ denotes the time interval between the current time instant $t$ and the previous time instant. The cargo is assumed to interact with a kinesin at time $t$ in the same mode as at time $t-\Delta t$. Note that there are three different modes of interaction between the cargo and a kinesin as described in figure 2.

Next, the values of three variables $\left(x_{\mathrm{c}}, x_{\mathrm{n}}\right.$ and $\left.F_{\mathrm{c}}\right)$ are calculated at the current time $t$ using (1) (which provides two relations) and equation (10) (which relates $x_{\mathrm{c}}(t)$ and $F_{\mathrm{c}}(t)$ ).

Next, the newly calculated variables are used to ascertain if they satisfy the assumed interaction (e.g., slack or no slack, assisting or resisting loads, etc). If they do not satisfy the assumed interaction, then the interaction type is updated and the calculation for the dynamics is carried out again over that time step.

Next, the rate of the chemical state variable is obtained using equations (3) and (4). Then, the value of $\rho$ is calculated as $\rho(t)=\rho(t-\Delta t)+\frac{\mathrm{d} \rho}{\mathrm{d} t} \Delta t$. If $\rho(t)$ is between 1 and -1 , the chemical cycle has not completed. Thus, the heads of kinesin remains in their previous position. If $\rho(t)$ is larger than 1 (or less than -1 ), the time instant $\widetilde{t}$ when the chemical cycle is completed $(t-\Delta t \leq \widetilde{t} \leq t)$ is computed by capturing the instant when $\rho$ becomes 1 (or -1 ) through a linear interpolation. The positions and forces at the current time are also changed to the values corresponding to $\widetilde{t}$. At that instant the heads are assumed to change positions. Namely, the trailing head is instantaneously relocated to the next binding site because the diffusing of the head is assumed to occur instantaneously. Also, the forces are updated to the values corresponding to that instant and those new positions. This procedure is carried out at every time instant during the calculation.

\subsection{Parameter values}

Experimentally obtained in vitro steady state force-velocity curves [14] are used to determine $K_{\mathrm{n}}, k_{1 f}, k_{1 b, 0}, k_{2 f}, \kappa$ and $\Phi_{\mathrm{c}}$.
Table 1. Parameters of the model.

\begin{tabular}{lll}
\hline Parameter & Value & Units \\
\hline$K_{\mathrm{c}}$ & 0.346 & $\mathrm{pN} \mathrm{nm}^{-1}$ \\
$K_{\mathrm{n}}$ & 2.238 & $\mathrm{pN} \mathrm{nm}^{-1}$ \\
$k_{1 f}$ & 1.513 & $\mu \mathrm{M}^{-1} \mathrm{~s}^{-1}$ \\
$k_{1 b, 0}$ & 1.026 & $\mathrm{~s}^{-1}$ \\
$k_{2 f}$ & 98.972 & $\mathrm{~s}^{-1}$ \\
$L_{\mathrm{c}}$ & 40 & $\mathrm{~nm}$ \\
$\Phi_{\mathrm{c}}$ & 1.430 & $\mathrm{~nm}$ \\
$\kappa$ & 2.561 & $\mathrm{pN} \mathrm{nm}$ \\
\hline
\end{tabular}

Their values are shown in table 1 . The fit is achieved by the nonlinear least-squares fit toolbox in MatLab (MathWorks). The values of $K_{\mathrm{M}}$ and $k_{2 f}$ are similar to their values calculated from previous in vitro experiments [10, 14]. The length of the cargo linker is assumed to be $100 \mathrm{~nm}$ because it consists of 400-900 amino acid residues [8]. The length of the cargo linker projected along the microtubule is calculated as $40 \mathrm{~nm}$ for a cargo of $1 \mu \mathrm{m}$ radius by using the geometric relation provided in [56]. Since the friction exerted on the cargo by the fluid is not considerable in vitro, the motion of the cargo is very fast (and occurs between relatively long dwell times). Hence, the in vitro force-velocity curve is insufficient to determine the value of $K_{\mathrm{c}}$. Instead, $K_{\mathrm{c}}$ is determined such that the total stiffness between the cargo and the microtubule $\left(\frac{K_{\mathrm{n}} K_{\mathrm{c}}}{K_{\mathrm{n}}+K_{\mathrm{c}}}\right)$ matches values obtained experimentally (approximately $0.3 \mathrm{pN} \mathrm{nm}^{-1}$ ) [57]. Figure 6 shows that the model is able to predict the velocity of kinesin very well by using the identified parameters.

The stall force of kinesin can be obtained also by using the model. To do that, one can extrapolate the force-velocity curve given by the model beyond $6 \mathrm{pN}$. A polynomial extrapolation predicts the stall forces of $7.6 \mathrm{pN}$ and $6.1 \mathrm{pN}$ for ATP concentrations of $2 \mathrm{mM}$ and $5 \mu \mathrm{M}$ which are close to the values presented in [14].

\section{Results}

In most previous studies of kinesin transport, the effects of the properties of the fluid were not analyzed because they do not play a major role in vitro. In a highly viscoelastic fluid, however, the properties of the fluid can have significant influences on kinesin behavior. The model introduced in this paper is designed to account for these effects. Since the friction exerted by the fluid decreases with the size of the cargo, a nano-sized cargo may be too small to clearly highlight the effectiveness of the model. Hence, the radius of the cargo is selected as $1 \mu \mathrm{m}$ in this paper.

In this section, we analyze the motion of kinesin in fluids with various properties to reveal the effects of viscosity and elasticity. In addition, the response of kinesin to fluctuating loads is examined by using the ability of the model to predict transient dynamics. Notably, we observe that even if the magnitudes of the fluctuating loads are the same, the speed of the kinesin changes with the frequency of the load. 


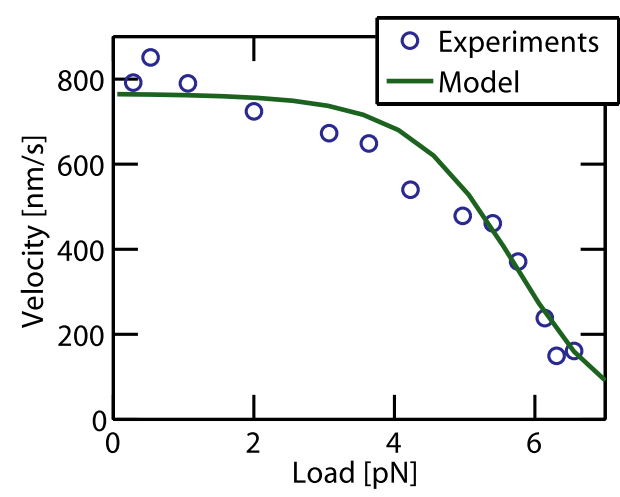

(a)

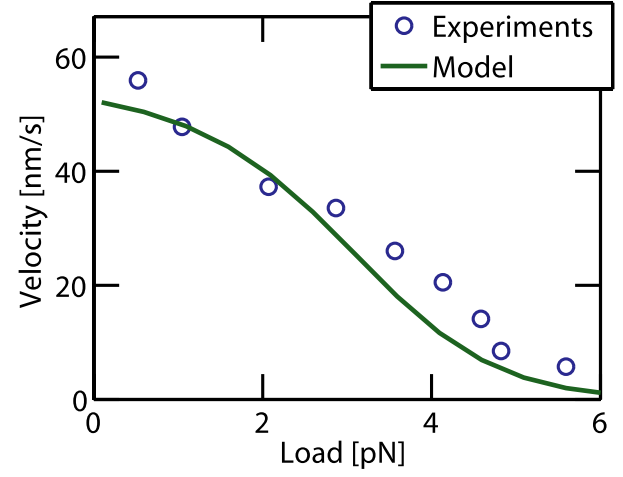

(b)

Figure 6. Experimental and predicted force-velocity curves for [ATP] $=2 \mathrm{mM}$ and $5 \mu \mathrm{M}$.

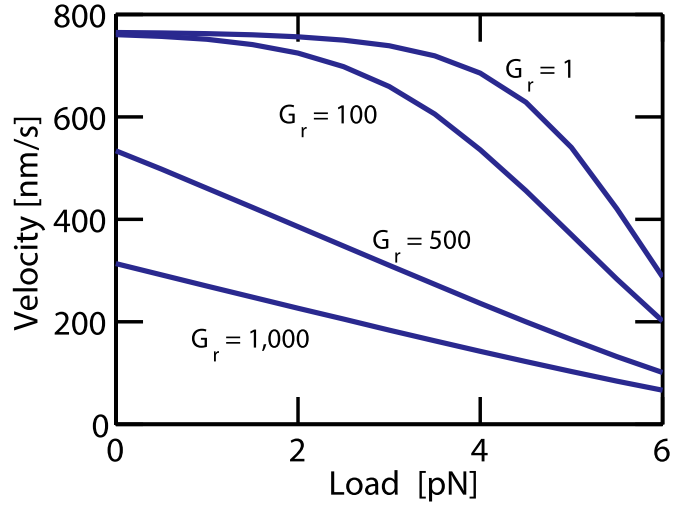

Figure 7. Effect of load on force-velocity curves for transport in various purely viscous fluids; $[\mathrm{ATP}]=2 \mathrm{mM}$.

\subsection{Effects of load and viscosity}

Many researchers have demonstrated that the speed of kinesin in vitro depends on the load $[14,52,58,59]$. If a single motor has no load, its in vitro velocity is about $800 \mathrm{~nm} \mathrm{~s}^{-1}$ when the concentration of ATP is $2 \mathrm{mM}$. The speed decreases to about $200-300 \mathrm{~nm} \mathrm{~s}^{-1}$ under a load of $6 \mathrm{pN}$. This speed is about $25 \%$ of the speed in the absence of a load. This large decrease indicates that the effect of the load is dominant in a fluid of low viscosity. However, the dependence of the velocity on the load becomes weak if the fluid is highly viscous. When viscosity is 1000 times higher than that of water, for example, the velocity under a high load $(6 \mathrm{pN})$ is just $50 \%$ of the velocity under no load. Another noticeable feature is the nonlinear relationship between load and velocity. For a fluid having low viscosity, kinesin maintains its highest velocity (for a given ATP concentration) even if the load is small $(0-3 \mathrm{pN})$. As the load increases, the velocity of kinesin starts to decrease. The rate of decrease keeps growing with the load. This nonlinear behavior of kinesin abates in a highly viscous fluid. Because kinesin significantly slows down when moving in fluids with high viscosity (even without a load), its force-velocity curve has an almost constant slope for high $G_{\mathrm{r}}$, as shown in figure 7 . Similarly, figure 8 shows that the effects of the load are more pronounced as $G_{\mathrm{r}}$ decreases.

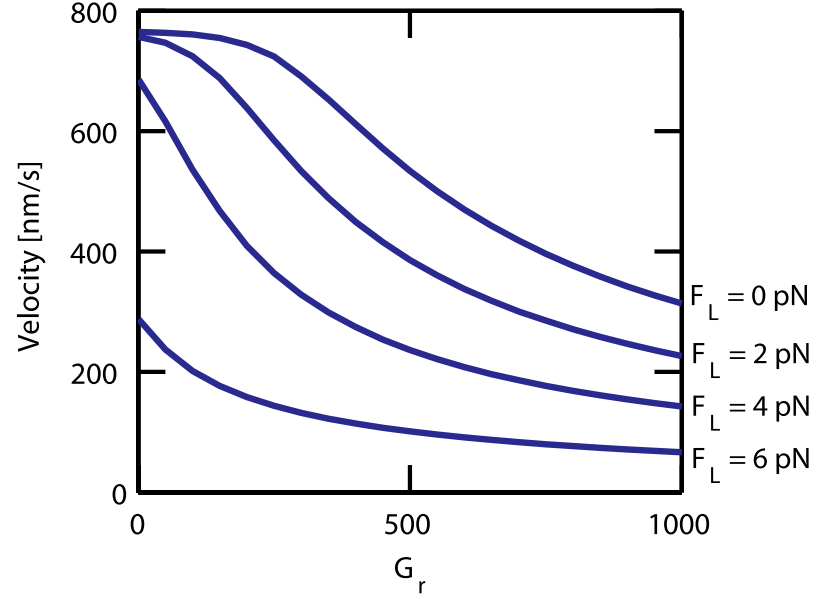

Figure 8. Effect of viscosity on velocity at various loads for transport in purely viscous fluids; $[\mathrm{ATP}]=2 \mathrm{mM}$.

\subsection{Effects of elasticity}

For viscoelastic fluids, it is difficult to observe the effects of elasticity when $G_{\mathrm{r}}$ is small. Fluids governed by the GLE have viscosity and elasticity that are proportional to $G_{\mathrm{r}}$. So, in the range of low $G_{\mathrm{r}}$, viscous and elastic force are weak. To observe the effect of elasticity, transport velocities in fluids with complex moduli corresponding to $G_{\mathrm{r}}$ higher than approximately 200 need to be compared. Thus, velocities of kinesin for various $\alpha$ were calculated for $G_{\mathrm{r}}$ values of 500 , 1000 and 1500.

The effect of the elastic component of the fluid force depends on $\alpha$, as shown in figure 9. Recall that a small $\alpha$ value corresponds to a low viscosity and a high elasticity. This property results in the rapid deceleration of the high initial velocity. The velocities can be examined by dividing the motion into three parts. At the beginning $(t<0.2 \mathrm{~s})$, the motion in the fluid with smaller $\alpha$ is faster because the viscous force is small while the elastic force is negligible (Part 1). As the kinesin moves forward $(0.2 \mathrm{~s}<t<3 \mathrm{~s})$, the intriguing relation between $\alpha$ and velocities is revealed. Since elasticity creates different forces over $\alpha$, the motor protein has high speeds at $\alpha=0.3-0.5$ (Part 2). For longer travel $(t>3 \mathrm{~s}$ ), the speed of kinesin is approximately proportional to $\alpha$ (Part 3). 


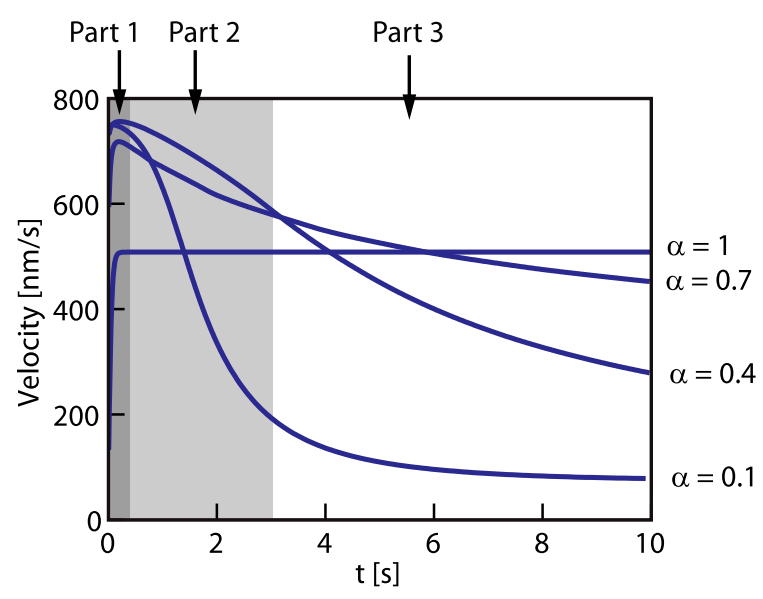

Figure 9. Velocity over time for $[\mathrm{ATP}]=2 \mathrm{mM}, G_{\mathrm{r}}=1000$, $\alpha=0.1,0.4,0.7$ and 1 . For $\alpha$ of 1 , the velocity does not change because there is no elastic force. For $\alpha$ less than 1, the velocity decreases over time due to the growth of the elastic component of the fluid force.

Part 3 may be inconsequential for kinesin transport for the following reasons. First, Part 3 can only take place if kinesin were to move for a very long time, which would require a run length much longer than that of a typical single kinesin. In vitro, a single kinesin moves about $0.8 \mu \mathrm{m}$, and that takes about $1 \mathrm{~s}$. After that, the kinesin detaches from the microtubule [59]. The run length depends on the load, but the order of magnitude of the time for a run length does not change significantly. If this run length and its duration are also valid for viscoelastic fluids, then Part 2 will dominate the motion of a single kinesin. Although the run length increases by some factor [60], kinesin may not have an opportunity to enter into Part 3 . Thus, if the steady state begins during Part 2 , then there will be no Part 3.

The elastic force exerted by the fluid on the cargo is related to the displacement of the cargo. A simple spring cannot be an accurate substitute for the elasticity of a complex fluid. However, a combination of a spring and a virtual reference is a physically feasible approximation for the dynamics. Figure 10 shows the conceptual explanation of the function of the elastic force on a molecular motor. When a cargo starts to move in the fluid, the elastic component of the fluid force is small and proportional to $\ell$, where $\ell$ refers to the distance between the cargo and the virtual reference. As the kinesin continues to move, the cargo and the virtual reference move also. The cargo moves faster than the virtual reference, and the difference in their velocities increases $\ell$, which in turn increases the elastic component of the fluid force. The increased force decreases the speed of kinesin until the motion

(a)

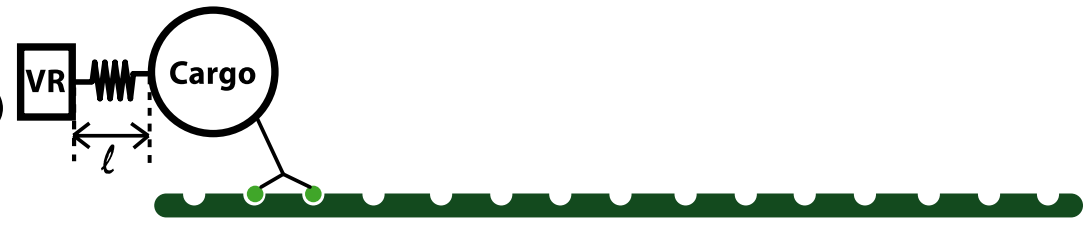

(b)

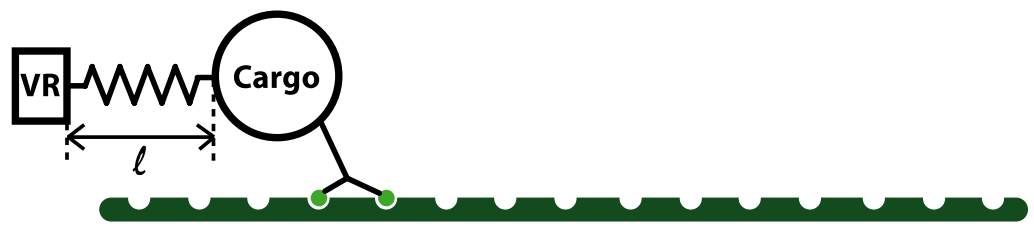

(c)

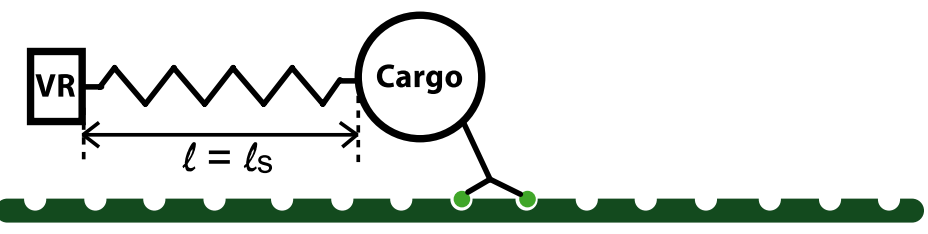

(d)

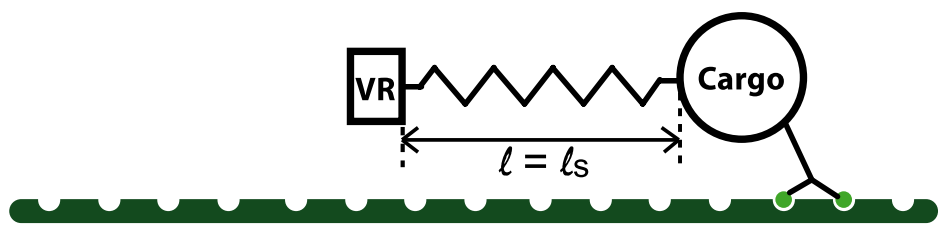

Figure 10. Behavior of the elastic component of the fluid force. VR denotes the virtual reference. When the motion of the cargo starts, $\ell$ is very small (a). $\ell$ increases as the kinesin moves (b). The steady state begins, and $\ell$ becomes $\ell_{\mathrm{s}}$ (c). In the steady state, $\ell$ remains constant $\ell=\ell_{\mathrm{s}}(\mathrm{d})$. 


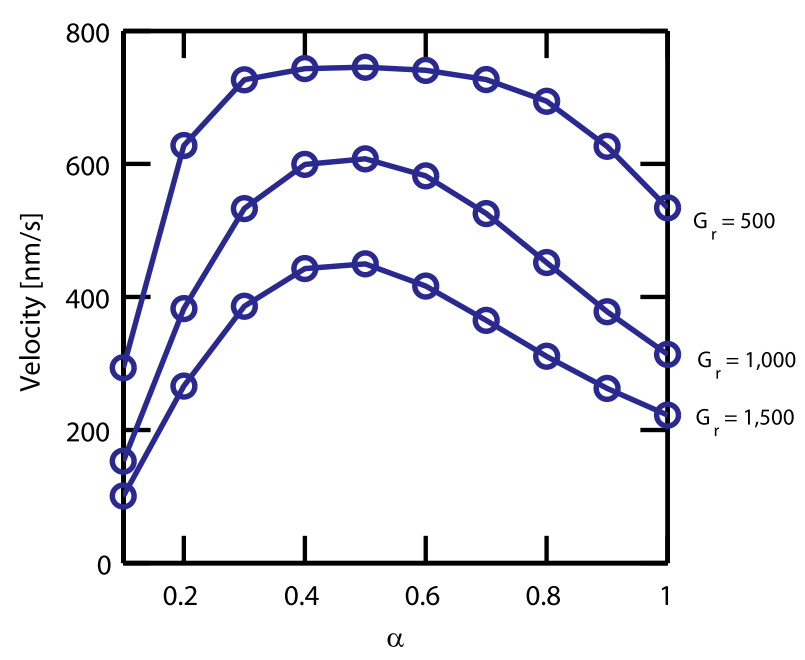

Figure 11. Steady state velocity versus $\alpha$ for $G_{\mathrm{r}}=500,1000$, and 1500 and $[\mathrm{ATP}]=2 \mathrm{mM}$.

of the cargo and the virtual reference are balanced. Then, $\ell$ does not change, and $\ell=\ell_{\mathrm{s}}$.

To capture the steady state motion, the memory function in the GLE has to be modified. The original memory function contains all past states. Thus, the continuously accumulating states in the function prohibit a steady state. However, for a long travel, states from the distant past have no effect on the current motion of kinesin. This is similar to the idea of a virtual reference. That means there exists a limited range of time in which the memory function varies. Beyond that, the memory function should have a constant value. This physically insightful approach, however, raises a challenge, namely that the current data about the cytoplasm do not supply too many clues about the steady states. Nevertheless, we can estimate the limited range from the experiments on the steady state motion of kinesin in the following way. In a cell, a cargo proceeds by pushing its way through polymers in the cytoplasm. In this motion, the key for establishing the limited range is the effective length, $\ell_{\mathrm{s}}$. This length indicates the distance over which one polymer affects the cargo. $\ell_{\mathrm{s}}$ depends on the size of the cargo and the length of the polymers. We assume that the average effect of the length of the polymers on $\ell_{\mathrm{S}}$ is the same regardless of the values of $\alpha$ and $G_{\mathrm{r}}$. If this assumption is applicable for a wide range of $G_{\mathrm{r}}$, the size of the cargo is the only parameter that determines $\ell_{\mathrm{s}}$. Thus, $\ell_{\mathrm{s}}$ is assumed to be proportional to the radius of the cargo $\left(\ell_{\mathrm{S}}=\beta r\right.$, and $\beta$ is constant over $G_{\mathrm{r}}$ and $\alpha$ ). In experiments with kinesin in an artificially mixed viscoelastic fluid ( $\alpha=0.51, G_{\mathrm{r}}=1590$ and $[\mathrm{ATP}]=1 \mathrm{mM})$, the steady state velocity of a single motor is about $370 \mathrm{~nm} \mathrm{~s}^{-1}$ [28]. By inserting this data into our model, the value of $\beta$ was calculated as 0.25 . With the concept of $\ell_{\mathrm{s}}$, the steady state velocities were obtained for $G_{\mathrm{r}}=500$, 1000, and 1500 and are shown in figure 11. These results support the idea that the steady state begins during Part 2 . The exciting result is that a single kinesin can transport cargoes faster in a viscoelastic fluid than in a purely viscous fluid, and it has a maximum velocity (for a given value of $G_{\mathrm{r}}$ ) when $\alpha$ is between 0.4 and 0.5 , as shown in figure 11 . This indicates that a ratio of approximately 0.5 between elasticity and viscosity builds the optimal circumstance for the fast transport (done by a single kinesin) in a highly viscoelastic fluid.

\subsection{Motion of vesicles in vivo}

Kinesins are involved in fast anterograde axonal transport. Their degraded intracellular transport capacity can cause neurodegenerative diseases such as Alzheimer's [61]. Thus, speed of kinesin is one of the most significant characteristics of the transport. The speed of kinesin in vivo is affected by the viscoelasticity of the cellular fluid. The characteristics of the viscoelastic fluid may lead to a slower or a faster transport.

The key parameters of the viscoelastic fluid are $\alpha$ and $G_{\mathrm{r}}$. Parameter $\alpha$ provides information regarding the ratio between viscosity and elasticity, and parameter $G_{\mathrm{r}}$ is an indicator for the magnitude of energy dissipation/viscosity/friction in the fluid. The model is able to predict the motion of kinesin if $\alpha$ or $G_{\mathrm{r}}$ are constant over frequency, as happens in some cytoplasm. Hill et al [29] measured the MSD of vesicles in the neurites of PC12 cells. They also tracked the motion of vesicles of radius $0.3-0.4 \mu \mathrm{m}$ which are transported by kinesin. They found an average velocity of $1250 \mathrm{~nm} \mathrm{~s}^{-1}$. The complex modulus of the cytoplasm of PC12 cells follows the FBM with the constant $\alpha$ and $G_{\mathrm{r}}\left(\alpha=0.75\right.$ and $\left.G_{\mathrm{r}}=2154\right)$, as shown in figure 12(a). Our model predicts that for the $G_{\mathrm{r}}$ value of 2154 (as in the PC12 cell), a cargo of radius $0.35 \mu \mathrm{m}$ (the size of vesicles used in experiments) has high velocities when $\alpha$ is $0.2-0.8$ (figure $12(\mathrm{~b})$ ). Though the predicted velocity is less than the observed velocity, $\alpha$ of 0.75 is the value for faster transport for the given $G_{\mathrm{r}}$ value. Note that the speed of vesicles in PC12 cells changes over time [29]. This speed is even higher than the maximum speed of a single kinesin in vitro. This considerably faster transport was also found by other researchers $[25,62,63]$. Thus, it is possible that the faster transport observed in vivo is due to other factors such as the cooperation of several kinesins. We also note that the values of $\alpha$ for several other cytoplasms vary over frequency [64]. Thus, the FBM with fixed $\alpha$ and $G_{\mathrm{r}}$ is not a general tool to predict motion of beads in any cytoplasm.

\subsection{Effects of the frequency of fluctuating loads}

The model provides accurate predictions for transient motions of kinesin. To observe the dynamics of kinesin under time varying loads, sinusoidally fluctuating loads are applied to the cargo. Of course, these fluctuations are transferred to the motor heads through the linkers. Thus, the rate of chemical reaction at the heads changes. The behavior of the cargo can be conceptually understood as the motion of a very small body connected to the ground by a spring and a dashpot. If the body experiences a fluctuating load, the amplitude of its fluctuating displacement is inversely proportional to the spring stiffness, the dashpot viscosity and the frequency of the load. Similar to that simple system, the fluctuations in the position of the cargo decrease as the viscoelasticity of the fluid increases, or the frequency of the load increases. As a result, the effects of the fluctuations vanish at high frequency, and the velocity-frequency curve flattens. In contrast, at 


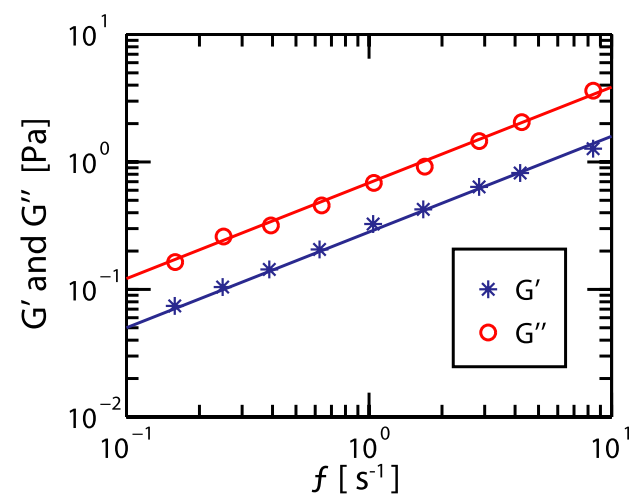

(a)

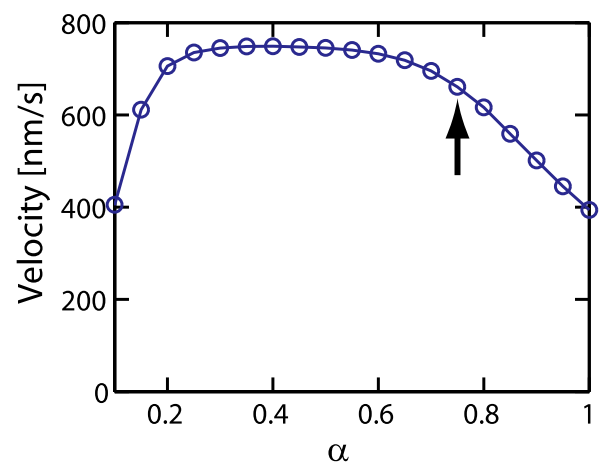

(b)

Figure 12. (a) The complex modulus of PC12 cell. Circles and stars are the experimental data, and lines represents the complex modulus of $\alpha=0.75$ and $G_{\mathrm{r}}=2154$. (b) The predicted velocity of the cargo of radius $0.35 \mu \mathrm{m}$ for $G_{\mathrm{r}}=2154$ and various $\alpha$. The arrow indicates the velocity corresponding to the property of PC12 cells.

lower frequencies, this curve has exponential variation. The approximate exponential curve is obtained using least mean square fitting to exact model predictions.

To discover the relationship between the motion of kinesin and the frequency of the load, two values are calculated: the changes in velocity $(\Delta v)$, and the frequency $\left(f_{\tau}\right)$ corresponding to $90 \%$ of the velocity difference. These values are shown in figure $13(\mathrm{a})$. For $G_{\mathrm{r}}=500$, the changes in velocity over frequency are large compared to other $G_{\mathrm{r}}$ values, and $\Delta v$ has its highest value at $\alpha=0.6$. The dependence of the velocity on frequency becomes weak when the viscoelasticity is strong, as shown in figure 13(b) (not unlike the case of a rigid body connected to the ground by a spring and dashpot). The value of $f_{\tau}$ also decreases over $G_{\mathrm{r}}$. The velocity for high $G_{\mathrm{r}}$ saturates at a relatively low frequency because viscoelasticity accelerates the saturation of the velocity-frequency curve, as shown in figure 13(c). This result indicates that kinesin is more sensitive to a fluctuating load in a fluid with low viscoelasticity than in a fluid with high viscoelasticity.

\section{Conclusions}

The newly developed model for the motion of kinesin provides solutions for several restrictions of existing models. By regarding a kinesin molecule as a structure composed of linearly elastic elements, it is possible to capture its transient and steady state dynamics. This approach also enables the prediction of the response of molecular motors under time varying loads. For a sinusoidally fluctuating load, the speed of kinesin changes with the frequency of the load. However, the effects of the fluctuation disappear at high frequency.

To describe the transport done by kinesin in a viscoelastic fluid, the GLE was applied to the model. The GLE was converted into a form which is suitable for calculating displacements with previous forces. This method is also useful for analyzing movements of other motor proteins in the presence of subdiffusion.

The cytoplasms is a highly viscoelastic fluid. We focused on the effect of large viscosity and elasticity on transport by molecular motor. Fluids having viscosity comparable with that of water do not have significant effects on the motion of kinesin. However, highly viscous fluids decrease the speed of kinesin to a quarter of its velocity in water. Moreover, the high viscosity also influences the shape of the force-velocity curve. While the curve for a fluid with low viscosity has a region where velocities are almost constant, the presence of large viscosity excludes that behavior.

To observe the effect of elasticity, the velocity of kinesin was calculated for various $\alpha$. Due to the complicated features of the complex modulus in subdiffusion, the viscoelastic properties were carefully designed. For fluids exhibiting subdiffusion, viscosity and elasticity cannot be changed individually. It would be great if a fluid of small $\alpha$ would have a lower viscosity and higher elasticity than another fluid of high $\alpha$. However, it is unavoidable that this relation is not followed in some frequency range for fluids following FBM. This problematic frequency range was reduced by a carefully selected reference frequency and by choosing the viscosity as the reference value for the magnitude of viscoelasticity. However, for a pure FBM, the elastic component of the fluid force grows as a motor walks. As a result, the $\alpha$ value (for the maximum instantaneous velocity) changes over time. Initially, $\alpha$ corresponding to the maximum velocity is 0.1 . If a steady state analysis is pursued, the value continuously increases over time.

Experimental data obtained through passive microrheology provides only limited information regarding the steady motion of a particle under a constant force in a cellular fluids. To address this issue, we used experimental steady state velocity values for cargoes transported by kinesin in a viscoelastic fluid and made an assumption regarding the effective length of the memory function. This approach predicted that kinesin has a maximum steady state velocity when $\alpha$ is $0.4-0.5$. Experimental research could verify this result. Also, in vivo experiments could directly measure the required distance for a particle to reach a steady state velocity under a constant force. Moreover, the fundamental equation used in this paper is available for the FBM having a single fractional number. This approximation is well suited for 


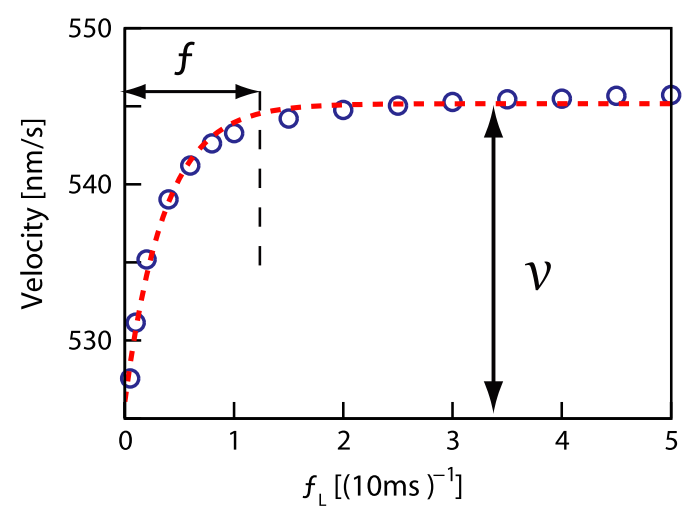

(a)

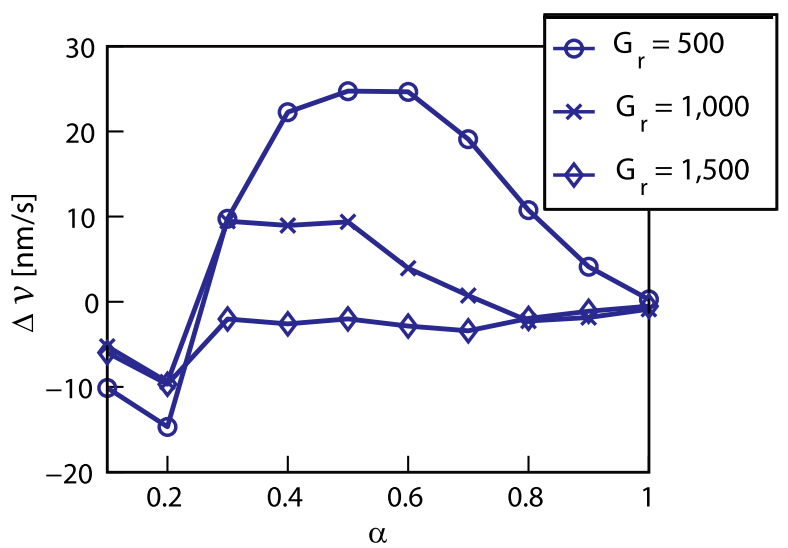

(b)

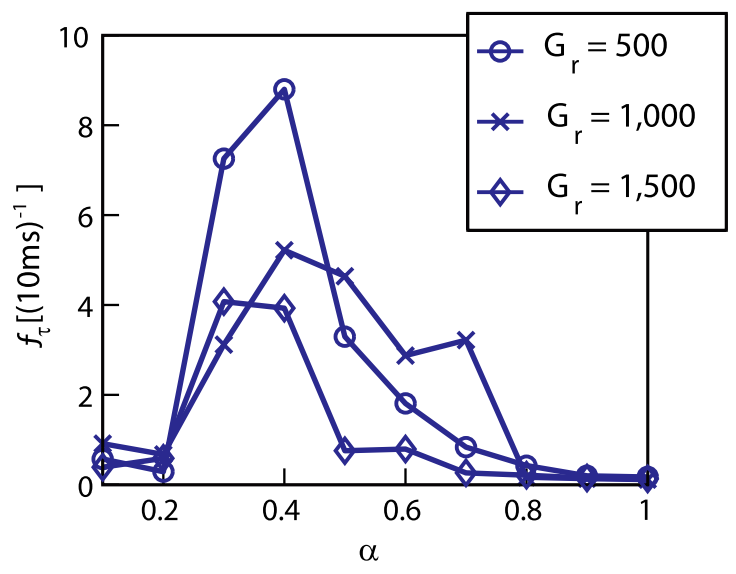

(c)

Figure 13. The response of kinesin to a load of

$F_{\mathrm{L}}(t)=2-2 \sin \left[\left(2 \pi f_{\mathrm{L}}\right) t\right] . f_{\mathrm{L}}$ denotes the frequency of the load. (a) Velocity-frequency curve for $\alpha=0.7, G_{\mathrm{r}}=500$. (b) $\Delta v$ versus. $\alpha$ for $G_{\mathrm{r}}=500,1000$, and 1500 . (c) $f_{\tau}$ versus $\alpha$ for $G_{\mathrm{r}}=500,1000$, and 1500 .

artificially created fluids and simulated cytoplasm [28, 47, 65]. However, it is not guaranteed that this relationship holds for every fluid. To cover more general cases, the memory function has to be extended to the level of describing the FBM with multiple fractional numbers. In addition, it is likely that a spatially varying complex modulus is present in vivo. That may require the memory function to transform continuously with the change in the complex modulus.

\section{Acknowledgments}

This work was supported by the National Science Foundation, CMMI Dynamical Systems Program. We would also like to express our gratitude to Professor Edgar Meyhöfer for insightful technical discussions of experimental techniques.

\section{References}

[1] Camalet S, Jülicher F and Prost J 1999 Self-organized beating and swimming of internally driven filaments Phys. Rev. Lett. 82 1590-3

[2] Grosh K, Zheng J, Zou Y, de Boer E and Nuttall A L 2004 High-frequency electromotile responses in the cochlea J. Acoust. Soc. Am. 115 2178-84

[3] Mandelkow E and Mandelkow E M 2002 Kinesin motors and disease Trends Cell Biol. 12 585-91

[4] Schliwa M and Woehlke G 2003 Molecular motors Nature 422 759-65

[5] Heck M M, Pereira A, Pesavento P, Yannoni Y, Spradling A C and Goldstein L S 1993 The kinesin-like protein klp61f is essential for mitosis in drosophila $J$. Cell Biol. 123 665-79

[6] Wordeman L and Mitchison T J 1995 Identification and partial characterization of mitotic centromere-associated kinesin, a kinesin-related protein that associates with centromeres during mitosis J. Cell Biol. 128 95-104

[7] Zhu C et al 2005 Functional analysis of human microtubule-based motor proteins, the kinesins and dyneins, in mitosis/cytokinesis using RNA interference Mol. Biol. Cell 16 3187-99

[8] Asbury C L, Fehr A N and Block S M 2003 Kinesin moves by an asymmetric hand-over-hand mechanism Science 302 2130-4

[9] Yildiz A, Tomishige M, Vale R D and Selvin P R 2004 Kinesin walks hand-over-hand Science 303 676-8

[10] Block S M, Asbury C L, Shaevitz J W and Lang M J 2003 Probing the kinesin reaction cycle with a $2 \mathrm{~d}$ optical force clamp Proc. Natl Acad. Sci. USA 1002351

[11] Hunt A J, Gittes F and Howard J 1994 The force exerted by a single kinesin molecule against a viscous load Biophys. $J$. $67766-81$

[12] Meyhöfer E and Howard J 1995 The force generated by a single kinesin molecule against an elastic load Proc. Natl Acad. Sci. USA 92 574-8

[13] Svoboda K and Block S M 1994 Force and velocity measured for single kinesin molecules Cell 77 773-84

[14] Visscher K, Schnitzer M J and Block S M 1999 Single kinesin molecules studied with a molecular force clamp Nature 400 184-9

[15] Astumian R D 1997 Thermodynamics and kinetics of a brownian motor Science 276 917-22

[16] Fisher M E and Kim Y C 2005 Kinesin crouches to sprint but resists pushing Proc. Natl Acad. Sci. USA 102 16209-14

[17] Fisher M E and Kolomeisky A B 1999 Molecular motors and the forces they exert Physica A 274 241-66

[18] Fisher M E and Kolomeisky A B 2001 Simple mechanochemistry describes the dynamics of kinesin molecules Proc. Natl Acad. Sci. USA 98 7748-53

[19] Fox R F and Choi M H 2001 Rectified brownian motion and kinesin motion along microtubules Phys. Rev. E 63051901

[20] Jülicher F, Ajdari A and Prost J 1997 Modeling molecular motors Rev. Mod. Phys. 69 1269-81 
[21] Klumpp S, Mielke A and Wald C 2001 Noise-induced transport of two coupled particles Phys. Rev. E 63031914

[22] Peskin C S and Oster G 1995 Coordinated hydrolysis explains the mechanical behavior of kinesin Biophys. $J$. 68 202S-11S

[23] Zeldovich K B, Joanny J F and Prost J 2005 Motor proteins transporting cargos Eur. Phys. J. E 17 155-63

[24] Shu Y G, Zhang X H, Ou-Yang Z C and Li M 2012 The neck linker of kinesin 1 seems optimally designed to approach the largest stepping velocity: a simulation study of an ideal model J. Phys.: Condens. Matter 24035105

[25] Kural C, Kim H, Syed S, Goshima G, Gelfand V I and Selvin P R 2005 Kinesin and dynein move a peroxisome in vivo: a tug-of-war or coordinated movement? Science 308 1469-72

[26] Ross J L, Ali M Y and Warshaw D M 2008 Cargo transport: molecular motors navigate a complex cytoskeleton Curr. Opin. Cell Biol. 20 41-7

[27] Holzwarth G, Bonin K and Hill D B 2002 Forces required of kinesin during processive transport through cytoplasm Biophys. J. 82 1784-90

[28] Gagliano J, Walb M, Blaker B, Macosko J C and Holzwarth G 2010 Kinesin velocity increases with the number of motors pulling against viscoelastic drag Eur. Biophys. J. 39 801-13

[29] Hill D B, Plaza M J, Bonin K and Holzwarth G 2004 Fast vesicle transport in pc12 neurites: velocities and forces Eur. Biophys. J. 33 623-32

[30] Shtridelman Y et al 2009 In vivo multimotor force-velocity curves by tracking and sizing sub-diffraction limited vesicles Cell Mol. Bioeng. 2 190-9

[31] Howard J 2001 Mechanics of Motor Proteins and the Cytoskeleton (Sunderland, MA: Sinauer Associates)

[32] Arigo M T and McKinley G H 1997 The effects of viscoelasticity on the transient motion of a sphere in a shear-thinning fluid J. Rheol. 41 103-28

[33] Frohlich H and Sack R 1946 Theory of the rheological properties of dispersions Proc. R. Soc. A 185 415-30

[34] King M J and Waters N D 1972 The unsteady motion of a sphere in an elastico-viscous liquid J. Phys. D: Appl. Phys. 5 141-50

[35] Oldroyd J G 1950 On the formulation of rheological equations of state Proc. R. Soc. A $200523-41$

[36] Thomas R H and Walters K 1966 The unsteady motion of a sphere in an elastico-viscous liquid Rheol. Acta 5 23-7

[37] Kou S C and Xie X S 2004 Generalized langevin equation with fractional gaussian noise: subdiffusion within a single protein molecule Phys. Rev. Lett. 93180603

[38] Lim S C and Muniandy S V 2002 Self-similar gaussian processes for modeling anomalous diffusion Phys. Rev. E 66021114

[39] Mason T G, Ganesan K, Van Zanten J H, Wirtz D and Kuo S C 1997 Particle tracking microrheology of complex fluids Phys. Rev. Lett. 79 3282-5

[40] Qian H 2003 Fractional Brownian Motion and Fractional Gaussian Noise (Berlin: Springer)

[41] Zwanzig R 2001 Nonequilibrium Statistical Mechanics (Oxford: Oxford University Press)

[42] Yamada S, Wirtz D and Kuo S C 2000 Mechanics of living cells measured by laser tracking microrheology Biophys. $J$. 78 1736-47

[43] Waigh T A 2005 Microrheology of complex fluids Rep. Prog. Phys. 685-742

[44] Mason T G 2000 Estimating the viscoelastic moduli of complex fluids using the generalized Stokes-Einstein equation Rheol. Acta 39 371-8
[45] Mason T G and Weitz D A 1995 Optical measurements of frequency-dependent linear viscoelastic moduli of complex fluids Phys. Rev. Lett. 74 1250-3

[46] Starrs L and Bartlett P 2003 One-and two-point micro-rheology of viscoelastic media J. Phys.: Condens. Matter 15 S251

[47] Shtridelman Y, Cahyuti T, Townsend B, DeWitt D and Macosko J C 2008 Force-velocity curves of motor proteins cooperating in vivo Cell Biochem. Biophys. 52 19-29

[48] Hendricks A G, Epureanu B I and Meyhöfer E 2008 Mechanistic mathematical model of kinesin under time and space fluctuating loads Nonlinear Dyn. 53 303-20

[49] Hendricks A G, Epureanu B I and Meyhöfer E 2009 Collective dynamics of kinesin Phys. Rev. E 79031929

[50] Hendricks A G, Epureanu B I and Meyhöfer E 2009 Cooperativity of multiple kinesin-1 motors mechanically coupled through a shared load Physica D 238 677-86

[51] Kou S C, Cherayil B J, Min W, Brian P and Xie X S 2005 Single-molecule Michaelis-Menten equations J. Phys. Chem. B 109 19068-81

[52] Carter N J and Cross R A 2005 Mechanics of the kinesin step Nature 435 308-12

[53] Holyst R et al 2009 Scaling form of viscosity at all length-scales in poly (ethylene glycol) solutions studied by fluorescence correlation spectroscopy and capillary electrophoresis Phys. Chem. Chem. Phys. 11 9025-32

[54] Szymanski J, Patkowski A, Wilk A, Garstecki P and Holyst R 2006 Diffusion and viscosity in a crowded environment: from nano-to macroscale J. Phys. Chem. B 110 25593-7

[55] Gennerich A and Schild D 2006 Finite-particle tracking reveals submicroscopic-size changes of mitochondria during transport in mitral cell dendrites Phys. Biol. 345

[56] Fehr A N, Gutiérrez-Medina B, Asbury C L and Block S M 2009 On the origin of kinesin limping Biophys. J. 97 1663-70

[57] Coppin C M, Finer J T, Spudich J A and Vale R D 1996 Detection of sub-8-nm movements of kinesin by high-resolution optical-trap microscopy Proc. Natl Acad. Sci. USA 931913

[58] Nishiyama M, Higuchi H and Yanagida T 2002 Chemomechanical coupling of the forward and backward steps of single kinesin molecules Nature Cell Biol. 4 790-7

[59] Schnitzer M J, Visscher K and Block S M 2000 Force production by single kinesin motors Nature Cell Biol. 2 718-23

[60] Thorn K S, Ubersax J A and Vale R D 2000 Engineering the processive run length of the kinesin motor J. Cell Biol. 151 1093-100

[61] Goldstein L S B 2001 Kinesin molecular motors: transport pathways, receptors, and human disease Proc. Natl Acad. Sci. USA 986999

[62] Ashkin A, Schütze K, Dziedzic J M, Euteneuer U and Schliwa M 1990 Force generation of organelle transport measured in vivo by an infrared laser trap Nature 348 346-8

[63] Kaether C, Skehel P and Dotti C G 2000 Axonal membrane proteins are transported in distinct carriers: a two-color video microscopy study in cultured hippocampal neurons Mol. Biol. Cell 11 1213-24

[64] Wirtz D 2009 Particle-tracking microrheology of living cells: principles and applications Ann. Rev. Biophys. 38 301-26

[65] Julicher F and Prost J 1997 Spontaneous oscillations of collective molecular motors Phys. Rev. Lett. 78 4510-3 